\title{
El software libre como herramienta para la enseñanza aprendizaje de soldadura eléctrica en el bachillerato técnico
}

\author{
Use of Free Software as a tool for Teaching Electric Welding Learning in the \\ Technical Baccalaureate
}

Juan Carlos Quishpe Cusi1. ', Christian Troya Torres. ${ }^{2}$, Fidel Parra Balza. ${ }^{3}$

\begin{abstract}
.
The present study aims to: develop a didactic guide using J CLIC software as a tool for the teaching of electric welding in technical high school, Quito Ecuador. Methodologically it is framed in a quantitative approach; the type of research is documentary and descriptive. As a population, we will work with the teachers and students of. Prof. Pedro Echeverría Terán School, specifically the students of Electric Welding of the third year of technical education. Methodologically, a review of documents, the survey of teachers and a sample of students were used to know the strategies used in the development of the subject and the knowledge they have of the proposed software. In the results highlights that a good number of students consider necessary to incorporate this type of tool into learning, because it can improve performance, it is also evident in the results that teachers despite having knowledge about ICT do not use in the process of teaching, as a conclusion it was reached that there are all the conditions to include Free Software as an educational tool that facilitates teaching learning of Electric Welding in the third year of technical baccalaureate of the educational institution Prof. Pedro Echeverría Terán located in the parish of Cumbayá -Lumbisí, Quito, Ecuador.
\end{abstract}

Keywords: Free Software, technological tool, teaching learning, JCLIC, technical baccalaureate, electric welding.

\section{Resumen.}

El presente estudio tiene como objetivo: desarrollar una guía didáctica utilizando el software $\mathbf{J}$ CLIC como herramienta para la enseñanza de soldadura eléctrica en bachillerato técnico, Quito

\footnotetext{
${ }^{1}$ Colegio Fiscal Profesor Pedro Echeverría Terán, Quito, juanc.quishpe@educacion.gob.ec

${ }^{2}$ Unidad Educativa Juan Montalvo. christian.troya@educacion.gob.ec

${ }^{3}$ Universidad Tecnológica Israel. fparra@uisrael.edu.ec
} 
Ecuador. Metodológicamente está enmarcado en un enfoque cuantitativo, el tipo de investigación es documental y descriptiva. Como población se trabajará con los docentes y alumnos de. Colegio Prof. Pedro Echeverría Terán, específicamente los cursantes de Soldadura Eléctrica del tercer año de educación técnica. Metodológicamente se utilizó revisión de documentos, la encuesta a los docentes y a una muestra de estudiantes para conocer las estrategias utilizadas en el desarrollo de la asignatura y el conocimiento que tienen del software propuesto. En los resultados destaca que un buen número de estudiantes considera necesario incorporar al aprendizaje este tipo de herramienta, debido a que puede mejorar el rendimiento, también se evidencia en los resultados que los docentes pese a tener conocimientos sobre las TIC no utilizan en el proceso de enseñanza, como conclusión se llegó a que existen todas las condiciones para incluir el Software Libre como herramienta educativa que facilite la enseñanza aprendizaje de Soldadura Eléctrica en el tercer año de bachillerato técnico. de la institución educativa Prof. Pedro Echeverría Terán ubicado en la parroquia de Cumbayá -Lumbisí, Quito, Ecuador.

Palabras claves: Software Libre, herramienta tecnológica, enseñanza aprendizaje, JCLIC, bachillerato técnico, soldadura eléctrica.

\section{Introducción}

En la actualidad existe una amplia y diversa oferta de herramientas informáticas. Se cuentan entre estos recursos los denominados hardware, que representan parte del equipo informático y periféricos acondicionados para satisfacer los cada vez más los exigentes gustos de los usuarios. Así también se tienen los programas y aplicaciones o software que al igual que los hardware se ofrecen siguiendo los requerimientos y necesidades de las personas e instituciones. Así bien, dentro de las TIC se pueden ubicar: la multimedia, el uso del Internet, páginas Web, blog, correo electrónico, Chat, video conferencias y los diversos softwares educativos, entre otros, (Castro, Guzmán, \& Casado, 2007).

Se requieren entonces nuevos modelos pedagógicos y experimentar otros escenarios que lleven a los actores de los sistemas educativos a asumir el reto de aprender con la tecnología y desde allí a través del empleo y la optimización de éstos innovar hasta convertirla en una herramienta cognitiva, que respondan a las nuevas realidades y necesidades del aula de clases (Colina, 2008).

Así que ante los retos que se plantean en la sociedad de la información y del conocimiento se requiere estructurar un nuevo patrón de escuela comprometido desde una óptica pedagógica, intercultural e inclusiva en la incorporación de propuestas que tomen en cuenta el suministro de software de bajo costo y software libre en las instituciones educativas que garantice el acceso a todos los componentes de la comunidad educativa, pudiendo así aminorar la brecha digital que se ha convertido en elemento discriminatorio producto de las diferencias de oportunidades entre los que tienen la posibilidad de acceso a las TIC y los que se les dificulta por no contar con los recursos económicos (Leiva \& Moreno, 2015). 
En la actualidad es muy frecuente que, en las instituciones de carácter educativo, empresariales y en los hogares el uso del software libre en lugar del software propietario. Son diversas los argumentos esgrimidos, entre las que se pueden destacar según lo expresado por (Gorostiaga, 2015) :

- No implica un costo de adquisición.

- El desarrollo de la creatividad, la solidaridad, la libertad y la exclusión como valores positivos, lo que constituye asimilar el software de una forma ética.

- Promueve el aprendizaje y la creación de material de forma colaborativa a través del apoyo mutuo.

Por otra parte, el Software Libre, lejos de propiciar al compartirse en un delito, resulta en una cooperación (Adell \& Bernabé, 2007). Pone en valor el conocimiento libre y abierto sobre la discriminación en el acceso (Valverde, 2007). Así mismo, (Cambero \& Llorente, 2007), hacen hincapié en la introducción de la cultura de la colaboración, no solo entre el alumnado sino entre el profesorado. Se puede decir que el Software Libre, favorece el desarrollo de una cultura tecnológica entre el alumnado que más allá de ser consumidores de software informático, aprenden su funcionamiento y se convierten en partícipe de su desarrollo.

Existen muchas aplicaciones disponibles para ser utilizadas en educación. Sin embargo, se ha encontrado con algunas limitaciones como: desconocimiento, confusión, resistencia y curva de aprendizaje, dificultad en la instalación y configuración, software disponible, falta de formación, acceso al código fuente, este último representa la facilidad de estudiar y modificar las aplicaciones exige un nivel de conocimiento al cual carecen muchos centros educativos (Adell \& Bernabé, 2007).

El contexto de estudio fue el colegio Prof. Pedro Echeverría Terán, ubicado en la parroquia de Cumbayá -Lumbisí, Quito, Ecuador; el cual fue creado hace 32 años, cuenta con 650 estudiantes y 30 docentes. Tienen entre sus objetivos la formación de Bachilleres Técnicos dispuestos a formar parte del mundo laboral y emprendedor. Específicamente se trabajó en el tercer año de bachillerato, en Soldadura Eléctrica. En la cual, por entrevistas realizadas al personal docente, desarrolla a través una pedagogía tradicional, de allí que se presenta la necesidad de aplicar mejoras en el proceso enseñanza aprendizaje, como lo es la introducción de las Tecnologías de Información y Comunicación con la finalidad de mejorar la motivación del estudiante en este tipo de conocimiento práctico y desarrollar en el mismo el autoaprendizaje.

De igual forma, como indican (Adell \& Bernabé, 2007) cobra importancia la inclusión de la programación en la adolescencia, etapa clave en las que el alumno requiere de modelos y ejemplos con los que probar y modificar.

Para resolver la situación antes citada, se planteó la introducción de software Libre, específicamente el software JCLIC; como instrumento útil para la enseñanza aprendizaje de Soldadura Eléctrica en los estudiantes de tercer año de bachillerato técnico del Colegio Prof. Pedro Echeverría Terán, ubicado en Quito, Ecuador. 
En ese sentido, la investigación se justifica ya que permitirá la introducción de contenidos teóricos a través del uso de una herramienta software libre JCLIC como preparación para el desarrollo de los elementos prácticos, lo que representa un hecho innovador en este tipo de contenido, además propicia el uso de la tecnología y el estudio independiente en otras de las especialidades del bachillerato técnico.

\section{Metodología}

En la actualidad existe una amplia y diversa oferta de herramientas informáticas. Se cuentan entre estos recursos los denominados hardware, que representan parte del equipo informático y periféricos acondicionados para satisfacer los cada vez más los exigentes gustos de los usuarios. Así también se tienen los programas y aplicaciones o software que al igual que los hardware se ofrecen siguiendo los requerimientos y necesidades de las personas e instituciones. Así bien, dentro de las TIC se pueden ubicar: la multimedia, el uso del Internet, páginas Web, blog, correo electrónico, Chat, video conferencias y los diversos softwares educativos, entre otros, (Castro, Guzmán, \& Casado, 2007).

Se requieren entonces nuevos modelos pedagógicos y experimentar otros escenarios que lleven a los actores de los sistemas educativos a asumir el reto de aprender con la tecnología y desde allí a través del empleo y la optimización de éstos innovar hasta convertirla en una herramienta cognitiva, que respondan a las nuevas realidades y necesidades del aula de clases (Colina, 2008).

Así que ante los retos que se plantean en la sociedad de la información y del conocimiento se requiere estructurar un nuevo patrón de escuela comprometido desde una óptica pedagógica, intercultural e inclusiva en la incorporación de propuestas que tomen en cuenta el suministro de software de bajo costo y software libre en las instituciones educativas que garantice el acceso a todos los componentes de la comunidad educativa, pudiendo así aminorar la brecha digital que se ha convertido en elemento discriminatorio producto de las diferencias de oportunidades entre los que tienen la posibilidad de acceso a las TIC y los que se les dificulta por no contar con los recursos económicos (Leiva \& Moreno, 2015).

En la actualidad es muy frecuente que, en las instituciones de carácter educativo, empresariales y en los hogares el uso del software libre en lugar del software propietario. Son diversas los argumentos esgrimidos, entre las que se pueden destacar según lo expresado por (Gorostiaga, 2015) :

- No implica un costo de adquisición.

- El desarrollo de la creatividad, la solidaridad, la libertad y la exclusión como valores positivos, lo que constituye asimilar el software de una forma ética.

- Promueve el aprendizaje y la creación de material de forma colaborativa a través del apoyo mutuo.

Por otra parte, el Software Libre, lejos de propiciar al compartirse en un delito, resulta en una cooperación (Adell \& Bernabé, 2007). Pone en valor el conocimiento libre y abierto sobre la 
discriminación en el acceso (Valverde, 2007). Así mismo, (Cambero \& Llorente, 2007), hacen hincapié en la introducción de la cultura de la colaboración, no solo entre el alumnado sino entre el profesorado. Se puede decir que el Software Libre, favorece el desarrollo de una cultura tecnológica entre el alumnado que más allá de ser consumidores de software informático, aprenden su funcionamiento y se convierten en partícipe de su desarrollo.

Existen muchas aplicaciones disponibles para ser utilizadas en educación. Sin embargo, se ha encontrado con algunas limitaciones como: desconocimiento, confusión, resistencia y curva de aprendizaje, dificultad en la instalación y configuración, software disponible, falta de formación, acceso al código fuente, este último representa la facilidad de estudiar y modificar las aplicaciones exige un nivel de conocimiento al cual carecen muchos centros educativos (Adell \& Bernabé, 2007).

El contexto de estudio fue el colegio Prof. Pedro Echeverría Terán, ubicado en la parroquia de Cumbayá -Lumbisí, Quito, Ecuador; el cual fue creado hace 32 años, cuenta con 650 estudiantes y 30 docentes. Tienen entre sus objetivos la formación de Bachilleres Técnicos dispuestos a formar parte del mundo laboral y emprendedor. Específicamente se trabajó en el tercer año de bachillerato, en Soldadura Eléctrica. En la cual, por entrevistas realizadas al personal docente, desarrolla a través una pedagogía tradicional, de allí que se presenta la necesidad de aplicar mejoras en el proceso enseñanza aprendizaje, como lo es la introducción de las Tecnologías de Información y Comunicación con la finalidad de mejorar la motivación del estudiante en este tipo de conocimiento práctico y desarrollar en el mismo el autoaprendizaje.

De igual forma, como indican (Adell \& Bernabé, 2007) cobra importancia la inclusión de la programación en la adolescencia, etapa clave en las que el alumno requiere de modelos y ejemplos con los que probar y modificar.

Para resolver la situación antes citada, se planteó la introducción de software Libre, específicamente el software JCLIC; como instrumento útil para la enseñanza aprendizaje de Soldadura Eléctrica en los estudiantes de tercer año de bachillerato técnico del Colegio Prof. Pedro Echeverría Terán, ubicado en Quito, Ecuador.

En ese sentido, la investigación se justifica ya que permitirá la introducción de contenidos teóricos a través del uso de una herramienta software libre JCLIC como preparación para el desarrollo de los elementos prácticos, lo que representa un hecho innovador en este tipo de contenido, además propicia el uso de la tecnología y el estudio independiente en otras de las especialidades del bachillerato técnico.

\section{Resultados}

\section{Utilidad de tic}

En el diagnóstico realizado al personal docente se encontró que el 100\% considera útil el uso de herramientas TIC durante el desarrollo de las clases de Soldadura Eléctrica. 


\section{Formas de dictar las clases}

El 100\% dicta sus clases en forma convencional y el $75 \%$ algunas veces han utilizado herramientas TIC en el aula. El 75\% nunca ha utilizado Software Libre como apoyo didáctico, sin embargo, el $100 \%$ ha participado en talleres de enseñanza de las TIC aplicadas a la educación. Por otra parte, es importante resaltar que el $100 \%$ está dispuesto a participar en el uso guía didáctica que facilite el aprendizaje de los contenidos que se abordan en Soldadura Eléctrica. Igualmente resaltar que esta disposición resultaría importante para el estudio, siendo un avance en el mismo en su proceder dentro del aula. Estos resultados coinciden con los obtenidos por (Angulo \& Valdés, 2011), sobre que los docentes poseen actitudes positivas respecto al uso de las TIC, lo cual se debe a la propagación de estas a todas las actividades de la vida diaria y el aumento de la facilidad de su uso.

En el diagnóstico realizado a los estudiantes se encontraron los siguientes resultados:

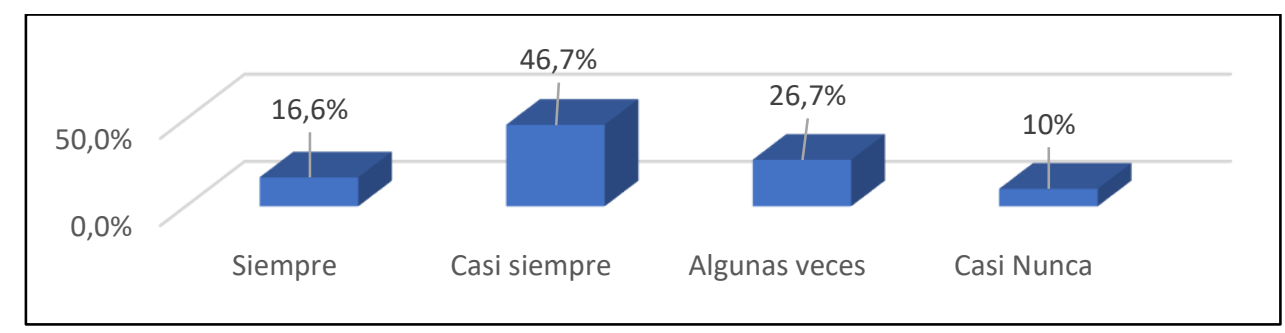

Figura 1. Opinión de los estudiantes a pregunta sobre si los contenidos de Soldadura Eléctrica los considera interesante.

Fuente: Elaboración propia

En respuesta a si los contenidos de Soldadura Eléctrica los considera interesantes, el $46.7 \%$ de los estudiantes consideran que casi siempre son interesantes, el 26.7\% algunas veces y solo el $16.6 \%$ siempre. Estos resultados significan que se deben utilizar otras herramientas para el proceso de enseñanza a fin de que los mismos resulten más atractivos para el resto de los $36.7 \%$ de los estudiantes.



Figura 2. Opinión de los estudiantes sobre si el docente utiliza herramientas para enseñar en forma fácil Soldadura Eléctrica.

Fuente: Elaboración propia 
Para la pregunta si el docente utiliza herramientas para enseñar en forma fácil Soldadura Eléctrica, se encontró que el 53\% considera que casi nunca, el $40 \%$ algunas veces y el $7 \%$ casi siempre. De aquí que resulta interesante la incorporación de nuevas herramientas, como lo es el Software Libre, que produzca en el estudiante un aprendizaje significativo. Como lo señala (Hermosa, 2015), el progreso educativo ha sido más atractivo, gracias a la adecuación del progreso tecnológico en este campo.

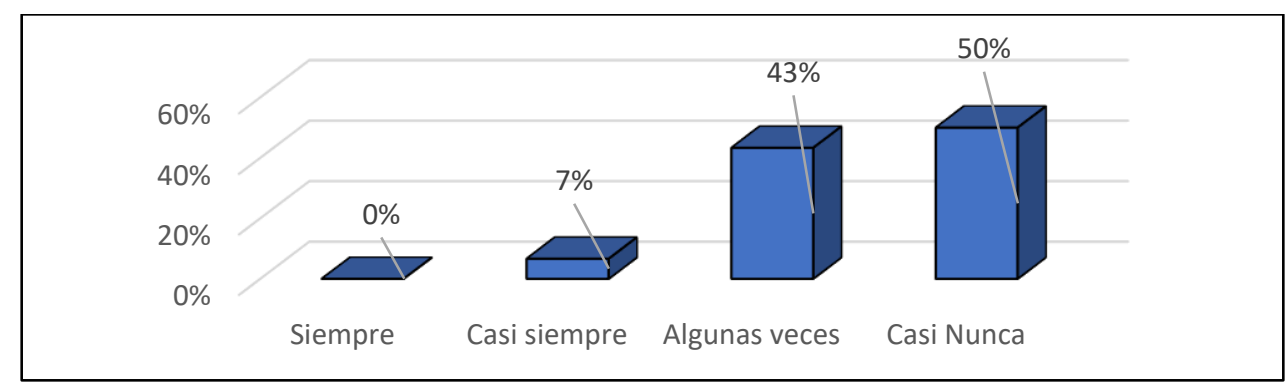

Figura 3. Opinión de los estudiantes sobre si el docente demuestra una actitud innovadora en clases.

Fuente: Elaboración propia

Sobre la respuesta de que si el docente demuestra una actitud innovadora en clases se tiene que el $43 \%$ considera que algunas veces y el $50 \%$ casi nunca. Este resultado indica poca innovación dentro del aula de clases, lo que implica la necesidad de un cambio en el proceso que lleva a cabo el docente.

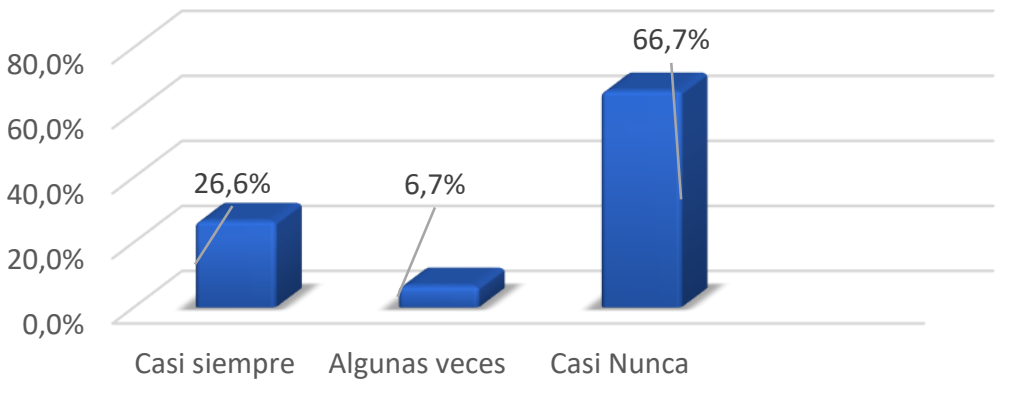

Figura 4. Opinión de los estudiantes sobre si el estudiante utiliza herramientas de Software Libre Fuente: Elaboración propia

En cuanto a la opinión de los estudiantes sobre si utiliza herramientas de Software Libre, se encontró que el $67 \%$ casi nunca las utiliza, el $27 \%$ casi siempre, solo un $7 \%$ algunas veces. Por otra parte, se tiene que el $100 \%$ de los mismos están dispuesto a que se incorpore un software libre como el JCLIC en el proceso enseñanza aprendizaje de Soldadura Eléctrica, lo que demuestra la disposición de los estudiantes a experimentar con nuevas herramientas que implique el uso de las TIC en el aula. Sobre lo anterior la (OEI, 2016) señala que los más jóvenes no pasaron por la sociedad estática que otros mayores si vivieron, por tanto, para ellos es muy normal todos los cambios y el aprendizaje continuo que se suscita en el campo educativo. 


\section{Validación de la propuesta a través de juicio de expertos}

Con la finalidad de validar la propuesta se sometió la misma a un juicio de tres expertos a los cuales se les suministró un instrumento que evaluaba la guía didáctica según los indicadores: calidad del lenguaje, precisión de instrucciones, secuencia de contenidos, apoyo gráfico, atractivo, sencillo y si facilita el aprendizaje. Los resultados de la validación de todos los expertos fueron de un promedio de aceptación de $92 \%$, lo cual se considera muy apropiado para ser implementado.

\section{Conclusiones}

- Los estudiantes consideran los contenidos que se imparten en Soldadura Eléctrica son interesantes, pero están dispuestos a que se introduzcan cambios a fin de que se experimenten nuevas formas de enseñar.

- Por otra parte, el docente manifiesta una actitud poco innovadora en la enseñanza de Soldadura Eléctrica, sin embargo, está dispuesto a incorporar las Tecnologías de Información y Comunicación en el aula, para lo cual tiene formación en el uso de estas.

- Así también se puede concluir que en la institución puede incluir el Software Libre como herramienta educativa que facilite la enseñanza aprendizaje de Soldadura Eléctrica ya que cuenta con la infraestructura y equipos para implementarla.

- Finalmente, la guía se realizó utilizando material visual, videos demostrativos, contenidos prácticos, actividades de autoevaluación para la enseñanza aprendizaje de Soldadura Eléctrica, utilizando software libre JCLIC, la cual fue validada por juicio de expertos, lo cual significa un aval para su implementación.

\section{Referencias Bibliográficas}

Adell, J., \& Bernabé, I. (2007). Software libre en educación. En J. Cambero, Tecnología Educativa (págs. 173-194). México: Mac Graw Hilll.

Angulo, J., \& Valdés, Á. (2011). Actitudes de Docentes hacia las Tecnologías de la Información y la Comunicación. XI Congreso Nacional de Investigación Educativa. Obregón, Sonora, México: Entornos Virtuales de Aprendizaje.

Arias, F. (2012). El proyecto de investigación: Introducción a la metodología científica. Caracas: Episteme.

Ausbel, \& Hanesian. (1990). Psicología educativa: un punto de vista cognitivo. México: Trillas.

Cambero, J., \& Llorente, M. (2007). Software Libre y sus posibilidades en la educación. Aula Interactiva, 48-55.

Castro, S., Guzmán, B., \& Casado, D. (2007). Las Tic en el proceso enseñanza aprendizaje. Laurus, Vol 13, num 23, pp.213-234.

Colina, L. (2008). Las Tic en los procesos de enseñanza-aprendizaje en la educación a distancia. Laurus, vol. 14, núm. 28, pp. 295-314. 
García , I., \& De la Cruz, G. (2014). Las guías didácticas: recurso necesario para el aprendizaje autónomo. Edumecentro, 162-175.

Gorostiaga, M. (2015). La integración de software libre en Centro de ESSO y Bachillerato de Bizkaia. Bilbao: Universidad Internacional de la Rioja.

Hermosa, P. (2015). Influencia de las tecnologías de información y comunicación (TIC) en el proceso enseñanza-aprendizaje: una mejora de las competencias digitales. Revista científica José María Córdoba , 121-132.

Hernández , R. (2014). Metodología de la Investigación. México: Mac Graw Hill.

Huerta, R., \& Luna, D. (2016). El software educativo JCLIC y su influencia en el desarrollo de las capacidades en el área de Historia, Geografía y Economía en los estudiantes del primer grado de secundaria de la I.E "Silvia Ruff" de Huari-2013. Lima. Perú: Tesis para optar al grado de maestría en Gestión e Innovación Educativa de la Universidad Católica Sedes Sapientiae.

JCLIC. (2018). Zona CLIC. Recuperado el 12 de Febrero de 2019, de https://clic.xtec.cat/legacy/es/jclic/

Leiva, J., \& Moreno, N. (2015). Recursos y estrategias educativas basado en el uso de harware de bajo costo y software libre: una perspectiva pedagógica intercultural. Eticanet, 38-50.

OEI. (25 de Mayo de 2016). Las TIC mejora de los procesos de enseñanza y aprendizaje. $\begin{array}{llllll}\text { Recuperado el de } 17 \text { junio de } & \text { de }\end{array}$ https://www.oei.es/historico/divulgacioncientifica/?Las-TIC-mejora-de-los-procesos-de

Oxgasa. (s.f.). Manual del Soldador.

Pérez , V., \& Fernández, J. (2005). Las tecnologías de la información y comunicación en la formación permanente del profesorado de educación física. Reifot, 8:1.

Piaget, J. (1975). Biología y Conocimiento. México: Editorial Siglo XXI.

Ramírez , M. (2016). Análisis de competencias adquiridas en el módulo de soldadura en estudiantes de tercero de bachillerato especialidad mecanizado y construcciones metálicas de la unidad educativa “Luis Tello”. Esmeralda, Ecuador: Tesis de Maestría de Pontificia Universidad Católica del Ecuador.

Rodríguez, R. (2009). Innovación metodológica docente en el marco del espacio de europeo de educación superior: algunas reflexiones desde los retos de la sociedad del conocimiento. XXI, Revista de Educación, 195-206.

UNESCO. (2005). Las tecnologías dela información y la comunicaciónen la enseñanza: manual para el docente. Montevideo: Uruguay: Ediciones Trilce.

Valverde, J. (2007). El software libre y las buenas prácticas educativas con TIC. Comunicación y pedagogía, 48-55.

Vidal, J. (2014). El Software “JCLIC” Para mejorar los niveles de comprensión lectora en los estudiantes de cuarto grado de educación primaria de la Institución Educativa Miguel Ángel Buonarroti del Distrito Porvenir, Trujillo, 2013 . Trujillo, Perú: Universidad Privada Atenor Orrego. 
Villalobos, V., Ávila, J., \& Olivares , S. (2016). Aprendizaje Basado en Problemas en química y el pensamiento crítico en secundaria. Revista Mexicana de Investigación Educativa, Vol 21 (69): 557-581.

Vizcarro, C., \& Juárez, E. (2007). La metodología del aprendizaje basado en problemas. Murcia. Vogt, P. (2007). Quantitative research methods for professionals. Boston: Pearson/Allyn and Bacon. 


\section{Para citar el artículo indexado.}

Quishpe Cusi, J. C., Troya Torres, C., \& Parra Balza, F. (2019). El software libre como herramienta para la enseñanza aprendizaje de soldadura eléctrica en el bachillerato técnico. Explorador Digital, 3(4), 55-65. https://doi.org/10.33262/exploradordigital.v3i4.915

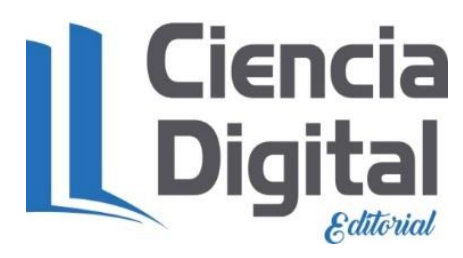

El artículo que se publica es de exclusiva responsabilidad de los autores y no necesariamente reflejan el pensamiento de la Revista Explorador Digital.

El articulo queda en propiedad de la revista y, por tanto, su publicación parcial y/o total en otro medio tiene que ser autorizado por el director o editor de la Revista Explorador Digital.

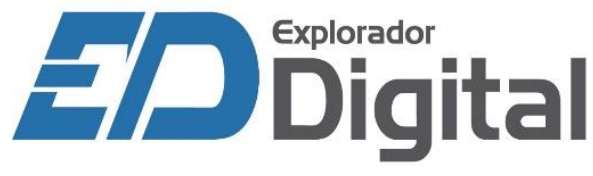

